\title{
INVESTIGAÇÃO EM SISTEMAS DE INFORMAÇÃO ORGANIZACIONAIS EM PORTUGAL: CARACTERIZAÇÃO DO PERÍODO DE 2004 A 2007
}

\author{
RESEARCH IN ORGANIZATIONAL INFORMATION SYSTEMS IN \\ PORTUGAL: CHARACTERIZATION OF THE PERIOD 2004 TO 2007
}

\author{
Ricardo Grilo \\ Instituto Politécnico de Leiria, Leiria, Portugal \\ ricardo.grilo@,ipleiria.pt \\ João Varajão \\ Universidade de Trás-os-Montes e Alto Douro, Vila Real, Portugal \\ Centro ALGORITMI, Guimarães, Portugal \\ jvarajao@utad.pt \\ Vitor Basto-Fernandes \\ Instituto Politécnico de Leiria, Leiria, Portugal \\ CIIC - Centro de Investigação em Informática e Comunicações, \\ Instituto Politécnico de Leiria, Leiria, Portugal \\ Vitor.fernandes@estg.ipleiria.pt \\ António Pereira \\ Instituto Politécnico de Leiria, Leiria, Portugal \\ CIIC - Centro de Investigação em Informática e Comunicações, \\ Instituto Politécnico de Leiria, Leiria, Portugal \\ apereira@estg.ipleiria.pt
}

\begin{abstract}
RESUMO
As tecnologias e sistemas de informação são um elemento central do desenvolvimento da Sociedade da Informação, encontrando-se actualmente múltiplas e variadas aplicações nas mais variadas áreas. Dada a sua importância, verifica-se uma grande diversidade de projectos de investigação neste campo, variando na sua natureza, objectivos, foco, e em muitos outros aspectos. Para acomodar as diferentes finalidades e características intrínsecas aos estudos nesta área, têm sido utilizados diferentes instrumentos de investigação, comummente organizados em epistemologias, abordagens, metodologias e técnicas. Procurando caracterizar a realidade da investigação em sistemas de informação organizacionais em Portugal no período de 2004 a 2007, realizou-se um trabalho de pesquisa e análise de teses e dissertações disponíveis em bibliotecas de universidades públicas portuguesas. Concretamente, identificaram-se os trabalhos de investigação realizados neste período e efectuou-se a respectiva análise, tendo em consideração a epistemologia subjacente, as abordagens adoptadas, as metodologias seguidas e as técnicas aplicadas. Neste artigo, após um enquadramento conceptual, são apresentados os principais resultados obtidos.
\end{abstract}

PALAVRAS-CHAVE: Sistemas de informação - Portugal. Metodologias científicas. Técnicas de pesquisas. Abordagens epistemológicas. 


\section{INTRODUÇÃO}

As tecnologias da informação (TI), no contexto do desenvolvimento competitivo das organizações, são cada vez mais relevantes, assumindo a investigação em sistemas de informação organizacionais (SI) o papel de motor do progresso, evitando que os aumentos exponenciais nas capacidades tecnológicas e nos níveis de investimento em TI nas organizações não sejam acompanhados por aumentos comparáveis na produtividade (VARAJÃO; AMARAL, 2000).

O despertar da Europa para a necessidade de maior investimento na investigação em ciência e tecnologia começou já no século XX, mas é em 2003, num estudo encomendado pela Comissão Europeia e presidido pelo ministro português Mariano Gago, que fica patente esta preocupação (EUROPEAN COMISSION, 2004). Como reflexo, a investigação em Portugal nos últimos anos, de uma forma geral, tem vindo a conhecer patamares de crescimento nunca antes verificados, resultado do esforço financeiro de apoio a programas de investigação e da abertura de projectos no seio das organizações. Contudo, uma análise sumária efectuada aos indicadores de investigação e desenvolvimento e à evolução do número de novos doutorados, permite concluir que Portugal se apresenta ainda num patamar de desenvolvimento inferior à média europeia, apesar do crescimento sustentado da despesa em investigação e desenvolvimento e do número de novos doutorados (DELLOITE, 2008). A investigação realizada no país é ainda em boa parte da responsabilidade das universidades públicas, através de cursos de mestrado e doutoramento.

Ao iniciar os trabalhos de um novo projecto, um investigador, tendo em consideração os objectivos propostos, procura identificar formas alternativas de os atingir. O percurso de investigação idealizado reflecte-se na aplicação de instrumentos de investigação, que tanto podem ser definidos devido à natureza do fenómeno, como devido à influência da comunidade de investigação em que o investigador está inserido. Na fase inicial do processo, um investigador normalmente equaciona diferentes abordagens para enfrentar o problema identificado e, dependendo da abordagem seleccionada, poderão existir diversas metodologias e técnicas que se afiguram mais adequadas para o processo de investigação. Por outras palavras, na condução de um projecto de investigação em sistemas de informação organizacionais, tal como acontece noutras áreas, um investigador necessita de definir diversos aspectos fundamentais que caracterizam todo o processo. Esses aspectos incluem a epistemologia subjacente ao trabalho de investigação, a abordagem seguida, a metodologia adoptada e o conjunto de técnicas utilizadas.

Neste artigo são apresentados os resultados de um estudo conduzido com a finalidade de caracterizar a realidade portuguesa da investigação em Sistemas de Informação Organizacionais (SI). De seguida, na secção 2, é efectuado um breve enquadramento conceptual. Na secção 3 é apresentado o processo de investigação definido. Na secção 4 são apresentados e discutidos os principais resultados obtidos. Finalmente, na secção 5, são tecidas algumas considerações finais.

\section{ENQUADRAMENTO CONCEPTUAL}

A produção de conhecimento através da investigação pode ser definida como uma actividade que contribui para a compreensão de um fenómeno (LAKATOS, 1978). Corresponde a um 
conjunto de actividades que a comunidade, onde se insere o projecto ou investigador, considera apropriada. $\mathrm{O}$ resultado obtido, para a maioria das comunidades de investigação deve corresponder a conhecimento que permita prever o comportamento, em determinados aspectos, do fenómeno. A epistemologia contempla não só a interpretação que se aplica ao resultado, mas também os pressupostos acerca do conhecimento e como pode este ser obtido (HIRSCHHEIM, 1992). Diferentes autores apresentam diferentes visões sobre epistemologia, contudo a posição partilhada da Association for Information Systems (AIS) identifica três vias principais para a interpretação de resultados, espelhadas nas classificações Positivista, Interpretativa e Ciência Crítica (CHUA, 1986; MYERS, 1997). A epistemologia Positivista baseia-se no pressuposto da existência de leis universais que governam os eventos sociais. O entendimento destas leis permite ao investigador descrever, prever e controlar o fenómeno (KIM, 2003). Neste meio é comum verificar que a comunidade apresenta grande preferência sobre dados observados e mensuráveis, que fundamentem os resultados como factos. A vertente Interpretativa, em contraste, procura compreender valores, crenças e significados do fenómeno, através da obtenção de análises profundas sobre actividades e experiências culturais (KIM, 2003). A Ciência Crítica procura apresentar uma compreensão crítica sobre o fenómeno, com o objectivo de sugerir uma transformação deste (WINBERG, 1997). A finalidade de um trabalho de investigação determinará se as respostas procuradas para um determinado conjunto de questões deverão ter fundamento em dados mensuráveis ou não. A investigação que visa analisar objectos ou acontecimentos observáveis e mensuráveis de alguma forma é apresentada como abordagem quantitativa. À investigação que visa ampliar o conhecimento da causa das coisas e do enquadramento de um fenómeno no mundo social, é atribuída designação de abordagem qualitativa. Para questões objectivas sobre ciências naturais, o investigador deve recorrer a instrumentos, técnicas e metodologias, que permitam obter dados quantitativos, estatísticos ou quantificáveis, criando investigação seguindo uma abordagem quantitativa. Em contraste, para dar resposta a questões de âmbito social, o investigador deverá aplicar instrumentos que permitam obter dados de natureza qualitativa, não quantificáveis, formando desta forma investigação com abordagem qualitativa.

A metodologia designa a lógica que estuda os métodos técnicos e científicos (MARTINS, 2000), assim como a arte de dirigir o espírito na investigação da verdade (PRIBERAM, 2009). Como resultado da revisão de literatura efectuada e da consulta de diversas fontes de dados, de que se destaca a AIS, verifica-se que a investigação em sistemas de informação apresenta como mais frequentes as seguintes metodologias: Action Research, Case Study, Ethnography, Design Research, Grounded Theory, Group Feedback, Experiments, Philosophical Research, Survey, Archival Research, Field Study, Opinion Research, Numeric Methods e Formal Methods. Neste artigo optou-se por manter sempre as designações no idioma original das referências consultadas, para facilitar a utilização futura, descritas a seguir:

Action Research apresenta quatro aspectos fundamentais: relevo dado aos participantes; colaboração através da participação; aquisição de conhecimento; e mudança social. O processo de investigação para conseguir estes fins é uma espiral de ciclos pesquisa-acção, que consiste em quatro fases principais: planeamento, acção, observação e reflexão (ZUBERSKERRIT, 1992; MASTERS, 1995);

Case Study requer uma completa observação, reconstrução e análise do fenómeno em estudo (ZONABEND, 1992). É vantajoso recorrer a Case Study quando o fenómeno assenta em 
procurar saber o "Como" ou o "Porquê", relativamente a um ou vários eventos contemporâneos, sobre os quais o investigador tem pouco ou nenhum controlo (YIN, 2003);

Ao adoptar Ethnography, o investigador insere-se durante um largo período de tempo no campo de acção, mergulhando profundamente na realidade e no ambiente sob estudo, procurando estudar o fenómeno no seu contexto social e cultural natural (LEWIS, 1985);

Fazer algo e avaliar os resultados é a base do modelo de processo da Design Research, funcionando como um ciclo em que o conhecimento é usado para criar trabalho, que por sua vez evolui para a formação de mais conhecimento (OWEN, 1997);

Grounded Theory procura desenvolver teoria fundada em dados que são sistematicamente recolhidos e analisados. Esta metodologia leva à descoberta, de forma indutiva, de conceitos teóricos acerca do fenómeno em estudo e simultaneamente permite fundamentar os conceitos através de dados ou de observação empírica (MARTIN; TURNER, 1986);

A aplicação de Group Feedback é um procedimento interactivo, onde os dados são recolhidos em diálogo com os informadores e, por sua vez, a interpretação dos resultados é feita pelas mesmas pessoas (DICK, 2005);

A metodologia Experiments apresenta como objectivo, medir os efeitos de $\mathrm{X}$ em $\mathrm{Y}$, controlando $\mathrm{X}$ e medindo $\mathrm{Y}$, mantendo ao mesmo tempo tudo o resto constante (BRISTOL, 2008);

A aplicação de Philosophical Research induz uma análise compreensiva e meticulosa sobre os objectivos críticos do estudo, sendo indicada para estudos teóricos;

Survey permite aceder a informação sobre o que faz e o que pensa um conjunto alvo de pessoas (BRYANT et al., 1980), através da aplicação de questionários que permitirão uma interpretação ou quantificação das respostas obtidas e, dessa forma, compreender melhor o fenómeno sob estudo;

Archival Research apresenta como foco da investigação o estudo de documentos, seguindo-se uma análise aos dados, que relatam sempre um facto já ocorrido (JENKINS; 1985);

Field Study recorre a técnicas de recolha não experimentais aplicadas sobre o ambiente natural do fenómeno, cuja aplicação implica a não manipulação de variáveis independentes nem o controlo de variáveis relevantes (BOUDREAU et al., 2001);

Opinion Research envolve a recolha de dados sobre atitudes, opiniões, impressões e crenças de indivíduos, conseguida através de meios de interrogação, como questionários, entrevistas ou outras técnicas. Permitindo testar hipóteses identificadas a priori, assim como a generalização de hipóteses, devido à sua abordagem iterativa (JENKINS, 1985);

Numeric Methods reflecte um processo determinístico e fechado onde todas as variáveis conhecidas, independentes e dependentes, são incluídas no modelo, não sendo possível alterar os valores das variáveis nem sendo necessário qualquer factor social (JENKINS. 1985); e Formal Methods consiste na aplicação de técnicas baseadas em métodos matemáticos que podem contribuir para a fiabilidade e robustez de um artefacto (HOLLOWAY, 1997). 
$\mathrm{Na}$ operacionalização das metodologias, os investigadores necessitam de recorrer a diferentes técnicas, que se referem a um conjunto de procedimentos metódicos que originam conjuntos de processos a realizar durante a investigação. Em SI, algumas das principais técnicas utilizadas são: DELPHI, Observation, Interview, Focus Group, Survey, Transcript Analysis, Measurement e Archival Research, a saber:

DELPHI recorre as especialistas sem os colocar fisicamente juntos (MASSER; FOLEY, 1987), procurando conseguir um consenso entre os participantes, sobre o assunto em discussão (MCCLAVE; BENSON, 1988; WAISSBLUTH; DE GORTARI, 1990; CHO et al., 1991) através de ciclos de resposta a questionários (FONTANA; FREY, 1994; AMAL, 2005);

Observation abrange todo o conjunto de operações que permitem submeter o fenómeno à verificação dos factos e, posteriormente, confrontá-lo com os dados observáveis (QUIVY; CAMPENHOUDT, 1998);

Interview permite obter informações de terceiros, relativamente às suas explicações ou razões a respeito das coisas precedentes, nomeadamente: o que sabem, crêem, esperam, sentem, desejam, pretendem fazer, fazem ou fizeram (SUASSUNA, 2008);

Focus Group é semelhante a entrevistas em grupo, capitalizando a comunicação entre vários participantes de forma a gerar informação. Apesar de as entrevistas em grupo serem por vezes usadas como uma forma rápida e conveniente de recolher dados de várias pessoas ao mesmo tempo, Focus Group recorre à interacção do grupo como parte da técnica (KITZINGER, 1995);

Ao adoptar Survey, o investigador procura respostas verbais ou escritas para questões ou declarações que permitirão recolher dados como preferências, expectativas, experiências vividas ou comportamentos privados do inquirido;

Transcript Analysis apresenta-se como uma forma de observar as comunicações criadas entre pessoas, através da análise de materiais com conteúdo de discussões, reuniões, entrevistas ou outro tipo de interacções, relativos a um tópico sob investigação (COOLICAN, 2004);

Measurement tem por objectivo determinar a influência de variáveis independentes sobre variáveis dependentes, identificando ou aplicando valores às variáveis independentes, para então concluir comportamentos ou implicações nos valores de variáveis dependentes (SCHWAB, 2004); e

Archival Research representa uma estratégia de investigação baseada na análise de documentos e registos administrativos, como fonte principal de dados, devido a estes serem os produtos que resultam das actividades diárias (SAUNDERS et al., 2007).

\section{PROCESSO DE INVESTIGAÇÃO}

Com vista a caracterizar a realidade portuguesa da investigação em SI, seguiu-se um processo sistemático de identificação, análise e classificação de trabalhos realizados nesta área, que decorreu no período de Outubro de 2007 a Maio de 2008. 
A investigação realizada em Portugal é em boa parte da responsabilidade das universidades públicas, através de cursos de mestrado e doutoramento. Nos últimos anos, a investigação em Portugal tem tido uma taxa de crescimento média anual (TCMA) de 8,7\%. Entre 1990 e 2006 o número de doutoramentos realizados ou reconhecidos por universidades portuguesas quase que quadruplicou de 337 para 1276. Entre 2000 e 2006 a TCMA baixa para 6,8\% (DELLOITE, 2008). Como reflexo, verifica-se um cada vez maior número de dissertações e teses, publicadas sob diversas formas pelas instituições de ensino, que pretendem não só contribuir para uma rede de conhecimento científico, mas também divulgar o trabalho científico da universidade.

Dadas as universidades serem os motores da investigação em sistemas de informação em Portugal, centrou-se nelas o esforço de pesquisa e identificação de trabalhos. Concretamente procuraram-se teses de doutoramento e dissertações de mestrado disponibilizadas em bibliotecas de universidades públicas. A decisão de focar o trabalho nestes repositórios deveuse ao facto de serem o local, até à data de realização do estudo, onde seria possível encontrar trabalhos desta natureza. O período analisado cobriu os anos de 2004, 2005, 2006 e 2007.

O grupo de ensino superior público universitário engloba 15 entidades (DGES, 2008), conforme é possível observar na Figura 1. Destes, no estudo realizado não foram analisados trabalhos de dois estabelecimentos de ensino por apresentarem características específicas: o caso da Universidade Aberta e do Instituto Superior de Ciências do Trabalho e da Empresa. A Universidade Aberta é uma instituição pública de ensino superior a distância (ABERTA, 2008). O ISCTE, Instituto Superior de Ciências do Trabalho e da Empresa, faz parte da rede de ensino superior público universitário, recebendo a consagração de instituto universitário não integrado, pelo Despacho Normativo n ${ }^{\circ} 11 / 1990$ (ISCTE, 2006). Esta definição coloca o instituto como estabelecimento de ensino superior universitário não integrado em universidade.

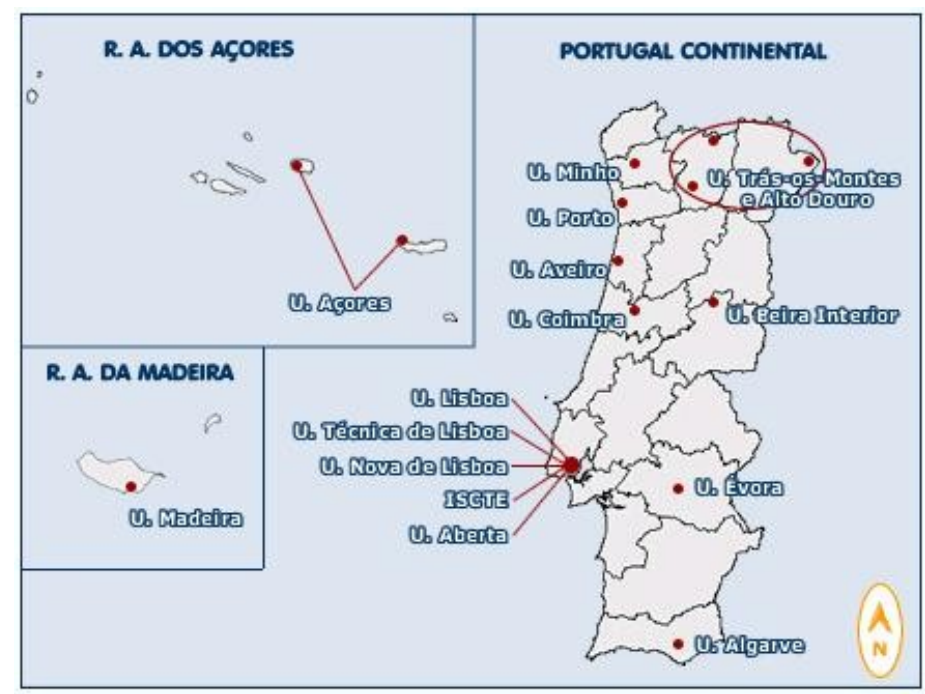

Figura 1 - Rede pública universitária de Portugal.

Fonte: (DGES, 2008)

Para a identificação dos trabalhos de investigação realizados nas universidades, recorreu-se a catálogos bibliográficos e repositórios de publicações. Tal permitiu agilizar o processo, dado que outros procedimentos alternativos se mostravam mais demorados, onerosos e de eficácia 
duvidosa. Por exemplo, a deslocação às bibliotecas de cada universidade poderia permitir maior controlo na identificação das obras, no entanto, para além de não permitir detectar os trabalhos em depósito ou requisitados na altura da visita, implicaria despender mais tempo e seria mais oneroso devido às deslocações necessárias. Outra possibilidade, o envio de um questionário aos departamentos de cada universidade relacionados com a área de SI, solicitando a indicação dos trabalhos realizados, apresentava um baixo nível de controlo sobre as respostas que se iriam obter.

Recorrendo aos catálogos bibliográficos disponibilizados por cada universidade, foi possível criar uma base de dados abrangendo as principais informações bibliográficas sobre todas as publicações do tipo dissertação ou tese. Estes foram identificados aplicando sempre o máximo de critérios de pesquisa (em função das características de cada catálogo bibliográfico), de acordo com o definido para o projecto: Assunto: Sistemas de Informação; Palavra: Tese ou dissertação; Anos: 2004 a 2007; Tipo de Documento: Monografia.

A identificação das publicações sobre sistemas de informação organizacionais, enquadradas nos critérios referidos, obrigou a um processo subsequente de análise sobre as publicações listadas pelas ferramentas de pesquisa. Com os constrangimentos encontrados nestas ferramentas, que não permitem parametrizar todas as variáveis do âmbito definido, foram identificadas inicialmente 5306 possíveis obras de interesse. A análise de cada estudo, através do resumo, índice e campos de catalogação, a eliminação de registos repetidos e a conformidade com os parâmetros estipulados, levaram a verificar que, das 5306 obras iniciais, apenas 169 eram efectivamente estudos da área de SI. Dado o domínio de temáticas considerado neste trabalho ter sido focado em sistemas de informação organizacionais, o número total de publicações alvo de caracterização acabou por ser reduzido para 89.

Cada trabalho identificado a que se teve acesso foi analisado em detalhe de modo a ser feita a respectiva caracterização de acordo com os instrumentos de investigação usados: identificando-se a abordagem adoptada, a metodologia seguida, as técnicas de investigação usadas na recolha de dados e a epistemologia subjacente na interpretação dos resultados conseguidos. A este trabalho de análise seguiu-se a organização e discussão de resultados.

\section{DISCUSSÃO DE RESULTADOS}

Do total de trabalhos identificado (89), apenas 25 se encontravam à data do estudo disponíveis online. $\mathrm{O}$ acesso aos restantes implicou um processo de requisição do estudo, presencial ou à distância através do protocolo Empréstimo Inter-Bibliotecas (EIB). Tal significa que apenas $28 \%$ dos estudos científicos do formato dissertação e tese, produzidos entre 2004 e 2007, relacionados com a área de SI, estavam disponíveis ao público através de plataformas Web, conforme é possível observar na figura 2. 


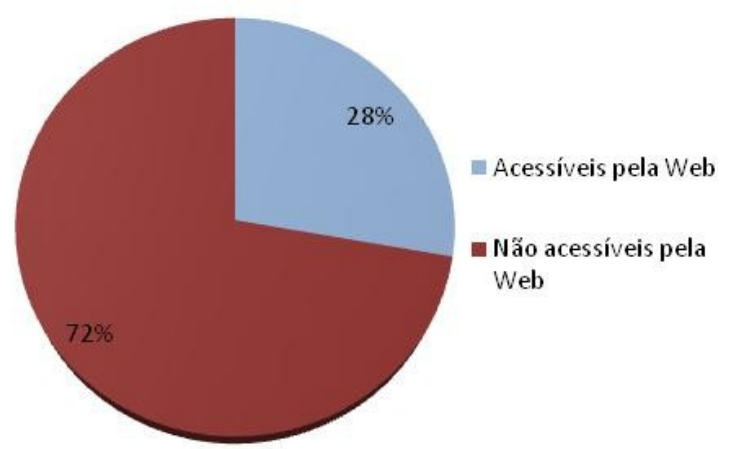

Figura 2 - Disponibilidade dos estudos na Internet.

Não foram analisados 16 estudos devido à indisponibilidade decorrente do empréstimo da obra (no caso de 7) ou à impossibilidade de deslocação às instalações da universidade que os detinha (no caso dos restantes 9). Assim, os resultados que são apresentados de seguida são baseados numa amostra de $82 \%$ do total de estudos em SI realizados em Portugal no período de 2004 a 2007, ou seja, 73 estudos.

Os dados recolhidos permitem verificar a distribuição do número de estudos em SI realizados ao longo do período de pesquisa (169 estudos no total). Na figura 3, verifica-se uma quebra no número de estudos realizados em 2007, o que provavelmente se deve ao facto das dissertações e teses desse ano ainda não estarem inseridas nos sistemas de gestão bibliográfica aquando da pesquisa efectuada.

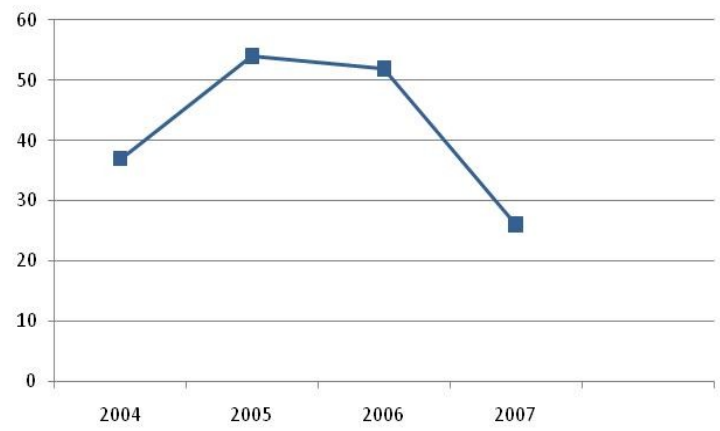

Figura 3 - Estudos de Sistemas de Informação de 2004 a 2007.

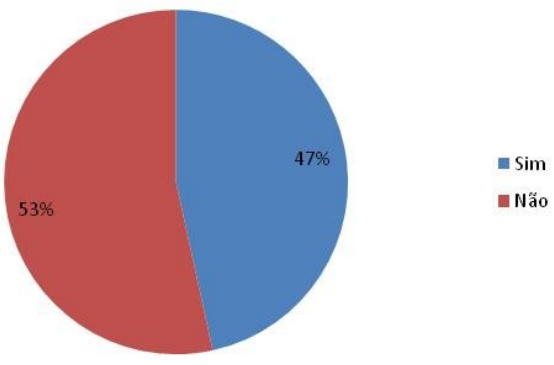

Figura 4 - Identificação explícita dos instrumentos de investigação adoptados nos estudos.

A caracterização dos 73 estudos em SI organizacionais recolhidos permite compreender melhor a investigação realizada. A figura 4 mostra que a maioria dos estudos não refere explicitamente, em nenhuma parte do documento, quais os instrumentos de investigação adoptados. É também possível observar na tabela 1 que apenas algumas das metodologias disponíveis para investigação em SI foram aplicadas em trabalhos de SI. Em particular, encontram-se em destaque as metodologias Action Research e Case Study. 


\begin{tabular}{lc}
\hline \multicolumn{1}{c}{ Metodologia } & $\mathbf{N}^{\mathbf{0}}$ estudos \\
\hline Action Research & 31 \\
Case Study & 28 \\
Survey & 6 \\
Archival Research & 4 \\
Grounded Theory & 3 \\
Design Research & 1 \\
\hline
\end{tabular}

Tabela 1 - Metodologias utilizadas nos estudos identificados.

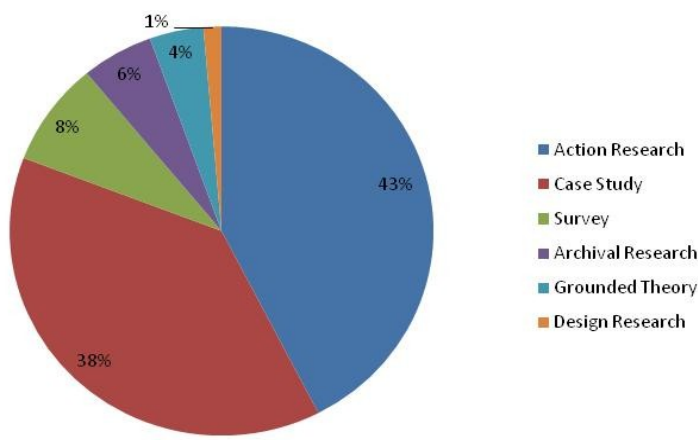

Figura 5 - Metodologias utilizadas na investigação de Sistemas de Informação Organizacionais em Portugal.

Os resultados indicam que no período em estudo, em Portugal, 43\% da investigação em SI foi desenvolvida recorrendo à Action Research (figura 4) e $42 \%$ dos trabalhos recorreram à técnica de investigação Observation, como ilustrado pela tabela 2 e figura 6.

\begin{tabular}{lc}
\hline \multicolumn{1}{c}{ Técnica } & $\mathbf{N}^{\mathbf{0}}$ estudos \\
\hline Observation & 54 \\
Archival Research & 36 \\
Interview & 23 \\
Survey & 11 \\
Measurement & 4 \\
DELPHI & 1 \\
\hline
\end{tabular}

Tabela 2 - Técnicas utilizadas nos estudos identificados.

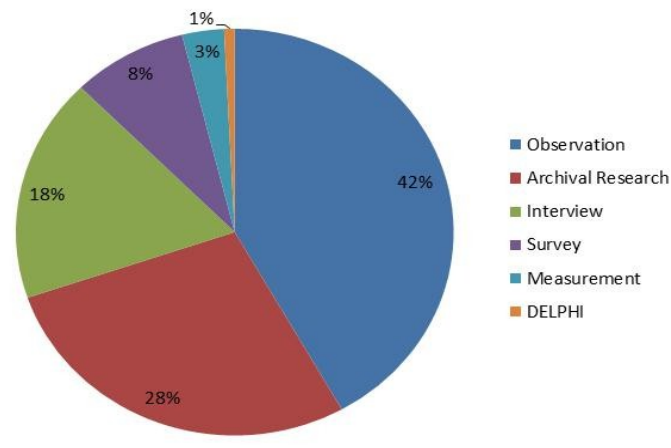

Figura 6 - Técnicas utilizadas na investigação de Sistemas de Informação Organizacionais em Portugal.

As técnicas de investigação mais aplicadas nos trabalhos analisados estão de acordo com as abordagens seguidas. Por se tratar de uma área onde os fenómenos são de difícil medição e têm uma grande intervenção social, as técnicas que os investigadores mais recorrem são as qualitativas. A figura 7 ilustra que $88 \%$ dos estudos são investigações do tipo qualitativo e os restantes $12 \%$ são do tipo quantitativo.

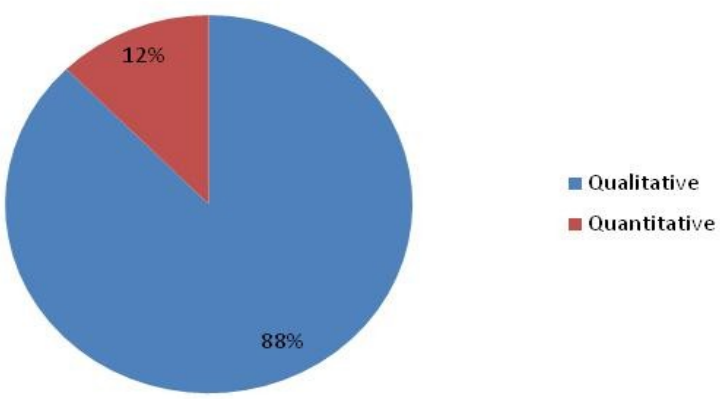

Figura 7 - Abordagens seguidas nos estudos identificados. 
Verifica-se também que os estudos são na larga maioria do tipo Qualitativo Interpretativo e em menor número, Qualitativo Positivista, havendo 9 estudos do tipo Quantitativo Positivista (ver tabela 3). A investigação em SI em Portugal possui em $81 \%$ dos casos uma discussão interpretativa dos resultados obtidos (ver figura 8).

\begin{tabular}{l|l|l}
\hline & Qualitativa & Quantitativa \\
\hline Positivista & 5 & 9 \\
\hline Interpretativa & 59 & - \\
\hline Ciência Crítica & 0 & - \\
\hline
\end{tabular}

Tabela 3 - Epistemologia e abordagens subjacentes aos estudos identificados.

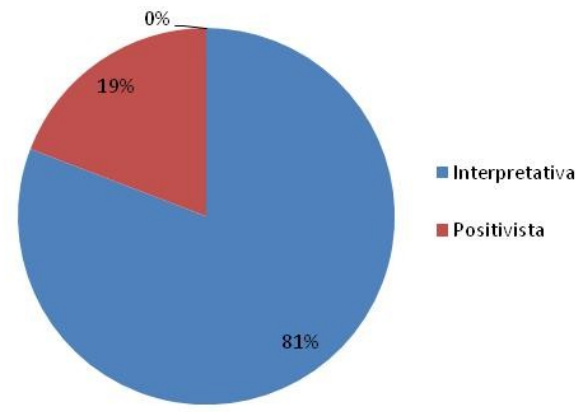

Figura 8 - Epistemologia subjacente à investigação de Sistemas de Informação Organizacionais em Portugal.

Com os dados obtidos é também possível caracterizar o relacionamento entre metodologias e as técnicas aplicadas nos estudos.

$\mathrm{Na}$ figura 9 encontra-se representada a relação existente entre metodologias e técnicas utilizadas nos estudos. Por exemplo, o relacionamento entre "Action Research" e "Interview" é caracterizado com um grau 3, significando que do total de estudos estruturados de acordo com Action Research, apenas 3 destes trabalhos aplicaram como técnica de recolha de dados Interview.

Nem todos os relacionamentos evidenciados pelos dados têm associados o mesmo grau de segurança de afirmação. A confiança nos relacionamentos associados a estudos Action Research ou Case Study, é bastante mais elevada, devido ao número de estudos analisados, do que relativamente a relacionamentos de estudos estruturados de acordo com outra metodologia. Por esse facto, a confiança relativa aos resultados obtidos com Action Research e Case Study é consideravelmente superior quando comparado com os outros casos. 


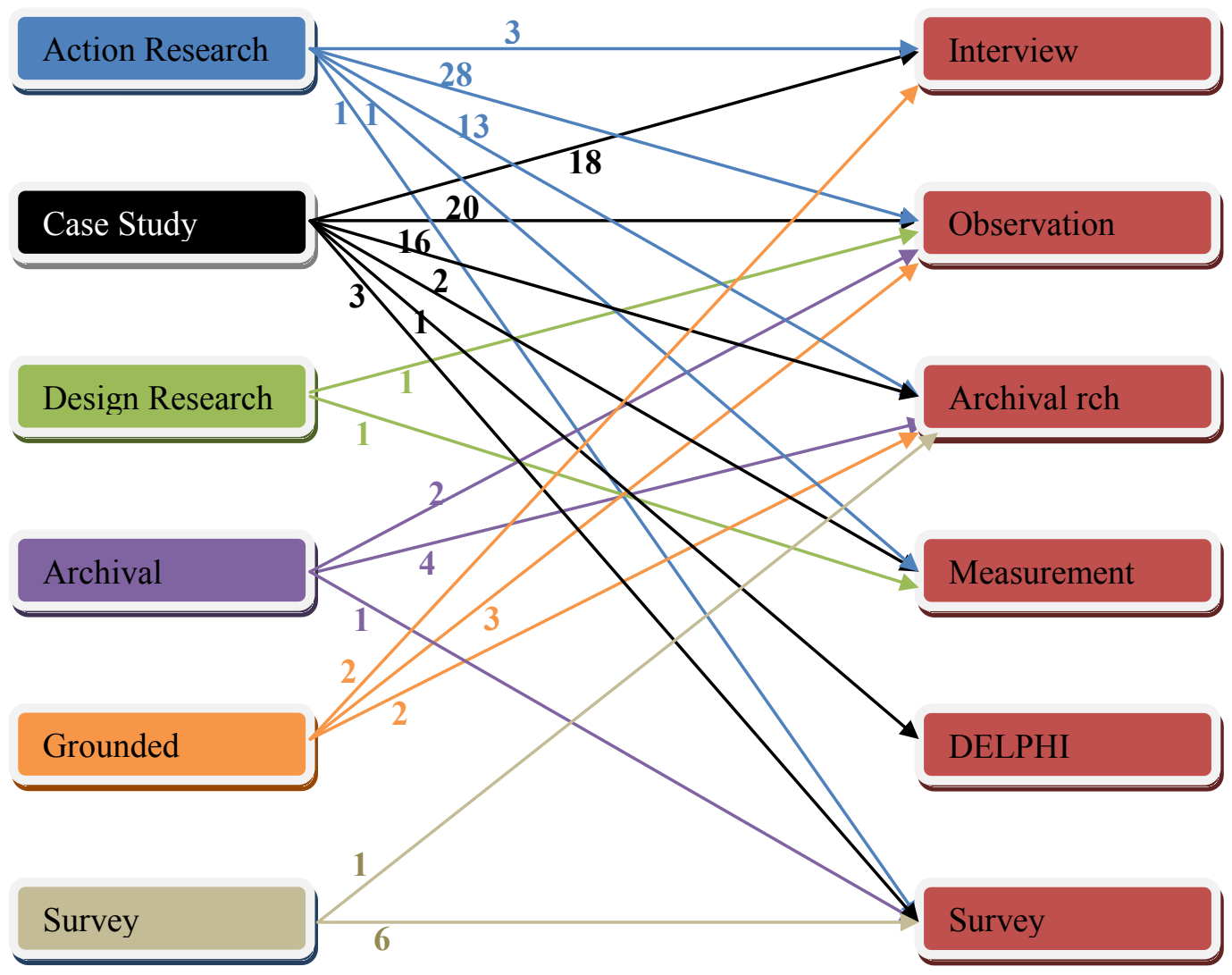

Figura 9 - Relação entre metodologias e técnicas em estudos de Sistemas de Informação Organizacionais.

\begin{tabular}{lllll}
\hline \multicolumn{1}{c}{ Perc. } & \multicolumn{3}{c}{ Metodologia } & \multicolumn{1}{c}{ Técnica } \\
\hline $\mathbf{9 0 , 3 \%}$ & dos estudos assentes em & Action Research & recorreram a & Observation \\
$\mathbf{4 1 , 9 \%}$ & dos estudos assentes em & Action Research & recorreram a & Archival Research \\
$\mathbf{9 , 7 \%}$ & dos estudos assentes em & Action Research & recorreram a & Interview \\
$\mathbf{3 , 2} \%$ & dos estudos assentes em & Action Research & recorreram a & Measurement \\
$\mathbf{3 , 2} \%$ & dos estudos assentes em & Action Research & recorreram a & Survey \\
$\mathbf{7 1 , 4 \%}$ & dos estudos assentes em & Case Study & recorreram a & Observation \\
$\mathbf{6 4 , 3 \%}$ & dos estudos assentes em & Case Study & recorreram a & Interview \\
$\mathbf{5 7 , 1 \%}$ & dos estudos assentes em & Case Study & recorreram a & Archival Research \\
$\mathbf{1 0 , 7 \%}$ & dos estudos assentes em & Case Study & recorreram a & Survey \\
$\mathbf{7 , 1 \%}$ & dos estudos assentes em & Case Study & recorreram a & Measurement \\
$\mathbf{3 , 6 \%}$ & dos estudos assentes em & Case Study & recorreram a & DELPHI \\
\hline
\end{tabular}

Tabela 4 - Síntese das técnicas aplicadas em metodologias (adoptadas em mais de dez estudos).

Na tabela 4 é feita uma síntese dos relacionamentos identificados. Verifica-se, por exemplo, que nos estudos que recorrem a Action Research, em cerca de $90 \%$ dos casos é usada a técnica Observation. 


\section{LIMITAÇÕES E TRABALHO FUTURO}

Os resultados apresentados neste artigo estão focados na informação que foi obtida directamente nos repositórios de universidades públicas à data de realização do trabalho. Tal significa, devido a algum atraso que tipicamente ocorre desde a finalização de um trabalho de investigação até ao momento em que fica disponível nas bibliotecas das universidades, que o ano de 2007 possivelmente não terá tido uma caracterização que reflicta em rigor os trabalhos desenvolvidos nesse ano. Dado hoje existirem novas ferramentas para divulgação e consulta de informação sobre trabalhos de natureza científica, transversais a diversas entidades de ensino superior (por exemplo, o Repositório Científico de Acesso Aberto de Portugal RCAAP), propõe-se como trabalho futuro a continuação do trabalho de investigação aqui apresentado, abarcando outras entidades para além das universidades públicas como, por exemplo, universidade privadas, centros e laboratórios de investigação, institutos politécnicos, etc. Interessante será também a comparação da realidade encontrada em Portugal com a realidade de outros países.

\section{CONCLUSÃO}

A análise dos trabalhos realizados em SI em Portugal entre 2004 e 2007 evidencia um leque de temas muito diversificado na área de SI. A maioria dos investigadores não refere instrumentos de investigação utilizados, sendo significativo o facto de $53 \%$ dos estudos analisados no âmbito deste trabalho não identificarem explicitamente quais os instrumentos de investigação adoptados. Fica a percepção de que boa parte dos investigadores da área desconhece, ou não valoriza, a importância da reflexão e adopção de um conjunto de ferramentas comprovadas que estruturem e dêem fundamento ao trabalho a realizar. A identificação dos instrumentos disponíveis, só por si, é uma tarefa morosa, devido à inexistência de referenciais que apresentem a um investigador que inicia o seu trabalho todo o leque de opções disponíveis para suportar o trabalho a realizar.

A expressiva diferença no número de estudos desenvolvidos segundo Action Research ou Case Study, comparativamente às restantes metodologias, evidencia a necessidade de estruturar os estudos de sistemas de informação organizacionais de forma a aproximar o trabalho à capacidade de compreender o relacionamento do fenómeno com o âmbito social que o rodeia. Esta necessidade é reforçada, pelo facto da operacionalização da realização dos estudos ser feita recorrendo maioritariamente à técnica Observation, em qualquer uma das metodologias adoptadas.

\section{REFERÊNCIAS}

ABERTA, Universidade. Missão. Disponível em: $<$ http://www.univ-ab.pt/ua/missao.php $>$. Acesso em: 03 dez. 2008.

AMAL, K. A. . Using the Delphi Technique to Search for Empirical Measures of Local Planning Agency Power. The Qualitative Report, v.10. n.4, 2005.

BOUDREAU, M. C. et al. . Validation in IS Research: A State-of-the-Art Assessment. MIS Quarterly, 25, 2001.

Enc. Bibli: R. Eletr. Bibliotecon. Ci. Inf., Florianópolis, n. esp., $2^{\circ}$ sem. 2010. ISSNe 1518-2924. 
BRISTOL, U. . Quantitative Methodologies. Disponível em: $<$ http://www.bris.ac.uk/Depts/DeafStudiesTeaching/dissert/Quantit $>$. Acesso em: 06 jun. 2008 .

BRYANT, B. et al. . Children and Minders. London, Grant McInryre, 1980.

CHO, Y. Y. et al. . A Delphi technology forecasting approach using a semi-Markov concept. Technological Forecasting and Social Change, 40, 1991.

CHUA, W. F. . Radical Developments in Accounting Though. The Accounting Review, 4, 1986.

EUROPEAN COMMISSION. Europe needs more scientists: EU blueprint for action, 2004.

Disponível em: $<$ http://www.publictechnology.net/content/837>.

COOLICAN, H. Research Methods and Statistics in Psychology, Hodder and Stoughton, 2004.

DELLOITE consultores. Estudo comparativode bolsas de doutoramento e pós doutoramento, 2008. . Fundação para a Ciência e Tecnologia, Portugal. Disponível em: < http://alfa.fct.mctes.pt/documentos/Relatorio_Deloitte_FCT_Bolsas_18_03_2008.pdf>.

DGES - Direccção-Geral do Ensino Superior. . Ensino Superior Público Universitário. MapaPubUniv.gif, 2008..

DICK, B. . Group feedback analysis. 2005. Disponível em: $<$ http://www.scu.edu.au/schools/gcm/ar/arp/gfa.html $>$. Acesso em 30 out. 2008.

FONTANA, A.; FREY, J. H.. Interviewing: The art of science. London, Sage, 1994.

HIRSCHHEIM, R. . Information Systems Epistemology: An Historical Perspective.

Information Systems Research: Issues, Methods and Practical Guidelines. R. Galliers. Oxford, Blackwell Scientific Publications: 28-60, 1992.

HOLLOWAY, C. M. . Why Engineers Should Consider Formal Methods. 16th Digital Avionics Systems Conference, 1997.

ISCTE - Instituto Superior de Ciências do Trabalho e da Empresa.. Resenha Histórica.

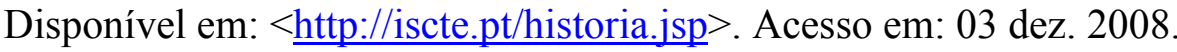

JENKINS, A. M. . Research Methodologies and MIS Research. Research Methods in Information Systems, s/n., 1985.

KIM, S. . Research Paradigms in Organizational Learning and Performance: Competing Modes of Inquiry. Information Technology, Learning, and Performance Journal, 21, 2003.

KITZINGER, J. Qualitative Research: Introducing focus groups. BMJ - British Medical Journal, 311, 1995.

LAKATOS, I. . The Methodology of Scientific Research Programmes. Cambridge, Cambridge University Press, 1978.

LEWIS, I. M. . Social Anthropology in Perspective. Cambridge, Cambridge University Press, 1985.

MARTIN, P. Y.; TURNER, B. A. . Grounded Theory and Organizational Research. The Journal of Applied Behavioral Science, s/n., 1986. 
MARTINS, G. A. Manual para elaboração de monografias e dissertações. São Paulo, Atlas, 2000 .

MASSER, I.; FOLEY, P. . Delphi revisited: Expert opinion in urban analysis. Urban Studies, v.24, n.3, 1987.

MASTERS, J. . The History of Action Research. Action Research Electronic Reader, 1995.

MCCLAVE, J. T.; BENSON, P. G.. Statistics for business and economics. San Francisco, Dellen, 1988.

MYERS, M. D. . Qualitative Research in Information Systems. MIS Quarterly, 21, 2, p. 241 242, 1997.

OWEN, C. . Design Research: Building the Knowledge Base. Journal of the Japanese Society for the Science of Design, 5, 1997.

PRIBERAM . Definição de Metodologia. Dicionário Priberam da Língua Portuguesa Priberam, Priberam Informática, S.A., 2009.

QUIVY, R.; CAMPENHOUDT, L. V. . Manual de Investigação em Ciências Sociais. Lisboa, Gradiva, 1998.

SAUNDERS, M. et al. . Research methods for business students. Pearson Education, 2007.

SCHWAB, D. P. Research Methods for Organizational Studies. Lawrence Erlbaum Associates, 2004.

SUASSUNA, D. Técnicas de Investigação Científica. Universidade de Brasília, 2008.

VARAJÃO, J.; AMARAL, L. . Outsourcing de Serviços de Sistemas de Informação: Origem e Evolução." Projecto MAPPA, Instituto de Informática, Ministério das Finanças, 2000.

WAISSBLUTH, M. DE GORTARI, A. . A methodology for science and technology planning based upon economic scenarios and Delphi techniques. Technological Forecasting and Social Change, v.37, n.4, 1990.

WINBERG, C. . How to Research and Evaluate. Cape Town, 1997.

YIN, R. K. . Case Study Research, Design and Methods. Newbury Park, Sage Publications, 2003.

ZONABEND, F. . The monograph in European ethnology. Current Sociology, 40, 1, 1992.

ZUBER-SKERRIT, O. Improving Learning and Teaching Through Action Learning and Action Research. HERDSA CONFERENCE University of Queensland, 1992. 


\begin{abstract}
Information technology and information systems are nuclear to Information Society development, nowadays in multiple and varied applications in various areas. Given its importance, there is a wide variety of research projects in this field, ranging in their nature, objectives, focus, and many other aspects. To accommodate the different purposes and characteristics intrinsic to studies in this area, have been using different research tools, commonly organized in epistemologies, approaches, methodologies and techniques. Trying to characterize the reality of research in organizational information systems in Portugal from 2004 to 2007, held a research and analysis of theses and dissertations available in libraries of Portuguese universities. Specifically, were identified the research undertaken in this period and made up their analysis, taking into account the underlying epistemology, the approaches, the methodologies and techniques applied. In this article, after a conceptual framework are presented the main results.
\end{abstract}

KEYWORDS: Information systems - Portugal. Scientific methodologies. Research techniques. Epistemological approaches.

Originais recebidos em: 15/04/2010

Aceito para publicação em: 10/10/2010 